\title{
APPROXIMATE ISOMETRIES ON FINITE DIMENSIONAL BANACH SPACES
}

\author{
BY \\ RICHARD D. BOURGIN(1)
}

\begin{abstract}
A map $T: E_{1} \rightarrow E_{2}$ ( $E_{1}, E_{2}$ Banach spaces) is an $\epsilon$-isometry if I $\|T(X)-T(Y)\|-\|X-Y\| \mid<\epsilon$ whenever $X, Y \in E_{1}$. The problem of uniformly approximating such maps by isometries was first raised by Hyers and Ulam in 1945 and subsequently studied for special infinite dimensional Banach spaces. This question is here broached for the class of finite dimensional Banach spaces. The only positive homogeneous candidate isometry $U$ approximating a given $\epsilon$-isometry $T$ is defined by the formal limit $U(X)=\lim _{r \rightarrow \infty} r^{-1} T(r X)$. It is shown that, whenever $T: E \rightarrow E$ is a surjective $\epsilon$-isometry and $E$ is a finite dimensional Banach space for which the set of extreme points of the unit ball is totally disconnected, then this limit exists. When $E=\ell_{1}^{k}\left(=k\right.$-dimensional $\left.\ell_{1}\right)$ a uniform bound of uniform approximation is obtained for surjective $\epsilon$-isometries by isometries; this bound varies linearly in $\epsilon$ and with $k^{3}$.
\end{abstract}

1. The form in which Hyers and Ulam [6] considered the $\epsilon$-isometry question is:

Does there exist a constant $K$ depending only on $\mathbf{E}_{1}$ and $\mathbf{E}_{2}$ with the following property: For each $\epsilon>0$ and surjective $\epsilon$-isometry $T: \mathbf{E}_{1} \rightarrow \mathbf{E}_{2}$ there is an isometry $U: \mathbf{E}_{1} \rightarrow \mathbf{E}_{2}$ with $\|T(X)-U(X)\| \leqslant K \epsilon$ for each $X$ in $\mathbf{E}_{1}$ ?

They observed that the assumption that $T$ be surjective is essential and answered (1.1) affirmatively when $\mathbf{E}_{1}=\mathbf{E}_{2}=$ Hilbert space [6]. D. G. Bourgin [2] showed more generally that (1.1) holds whenever $\mathbf{E}_{1}$ and $\mathbf{E}_{2}$ belong to a class of uniformly convex Banach spaces including the $\mathrm{L}_{p}(X, \Sigma, \mu)$ spaces $1<p<\infty$. A subsequent paper of Hyers and Ulam [7] gave a positive answer for $\mathbf{E}_{i}=\mathbf{C}\left(\mathrm{D}_{i}\right), i=1,2$ (the Banach spaces of continuous functions on the compact Hausdorff spaces $\mathrm{D}_{i}$ with the sup norm), provided $T$ is a homeomorphism. This study was continued

Received by the editors March 19, 1974.

AMS (MOS) subject classifications (1970). Primary 46A05; Secondary 46B99.

Key words and phrases. Approximate isometry, isometry, finite dimensional Banach space.

(1) Supported in part by the Research Foundation of the State University of New York. Copyright $\odot$ 1975, American Mathematical Society 
by Bourgin [3] who showed in particular that the continuity and 1-1 assumptions on $T$ could be lifted, thus providing a significant generalization of the classical theorem of Banach and Stone. For a general survey of these and related results see [4] and [9].

The modulus of uniform convexity for $\mathbf{L}_{p}$ and the maximal ideal structure of C(D) were central to the arguments of [2], [3] , and [7] and no general theory has emerged. In $\S 3,(1.1)$ is established for finite dimensional $l_{1}$ with the constant $K$ varying as the cube of the dimension. The validity of (1.1) in general is in doubt, and in this connection a possible line of attack for constructing a counterexample is given in $\S 4$. In particular it is shown there that if the constants $K$ in (1.1) for $\ell_{1}^{k}$ are necessarily unbounded as $k$ varies, then $\ell_{1}$ will be a space for which (1.1) fails (provided the isometries considered are positive homogeneous). The remainder of the first section delineates the bulk of the notation and includes some elementary observations while the second section establishes the existence of a candidate approximating isometry for a surjective $\epsilon$-isometry on certain finite dimensional spaces.

It is a pleasure to acknowledge the many useful conversations on the subject of this paper that I have had with my friend and colleague, Peter L. Renz. In particular Lemma 2.8 is due to him. It is impossible, however, to acknowledge adequately the encouragement and help I have received over the years, and in particular in regard to this study, from my teacher, colleague, friend and father, D. G. Bourgin.

Notation 1.2. (a) E (possibly with subscripts or primes) denotes a Banach space. The symbol dim $\mathbf{E}$ refers to the dimension of $\mathbf{E}$ and implicit in its use is the statement that $\mathbf{E}$ is finite dimensional.

(b) The points of $\mathrm{E}$ are denoted by the capital letters $V, W, X, Y$, and $Z$ (with or without subscripts, primes, or other secondary marks).

(c) For any $\alpha>0$ and $X$ in $\mathbf{E}$,

$$
\begin{aligned}
& \mathbf{B}(X, \alpha)=\{Y \text { in } \mathbf{E} \mid\|Y-X\|<\alpha\}, \\
& \mathbf{B}[X, \alpha]=\{Y \text { in } \mathbf{E} \mid\|Y-X\| \leqslant \alpha\} .
\end{aligned}
$$

(d) $U$ always denotes an isometry.

(e) $T$ always refers to an $\epsilon$-isometry such that $T(0)=0$.

(f) For a given $T: \mathbf{E}_{1} \rightarrow \mathrm{E}_{2}$, the function $S: \mathbf{E}_{2} \rightarrow \mathrm{E}_{1}$ will refer to any representative of $T^{-1}$. That is, $S$ is any map for which $T S(Y)=Y$ for each $Y$ in $\mathbf{E}_{\mathbf{2}}$.

Note that (1) $S$ is an $\epsilon$-isometry; and (2) if $X \in \mathrm{E}_{1}$ then $\|X-S T(X)\| \leqslant \epsilon$. ( $S$ is ' $\epsilon$-onto'. Cf. Definition 1.6.)

(g) The letter $\theta$ (with or without subscripts) will always be a number between -1 and 1 chosen so that the equation in which it first appears is true. 
For example, once $V$ and $W$ are known, $\|V+W\|-\|V\|=\theta\|W\|$ defines $\theta$.

(h) For each positive integer $k$ denote by $\ell_{1}^{k}$ the Banach space of $k$-tuples of real numbers with norm given by $\|X\|=\sum_{i=1}^{k}\left|x_{i}\right|$.

Suppose that $T: \mathbf{E}_{1} \rightarrow \mathbf{E}_{2}$ and that $U$ is a positive homogeneous isometry for which $\|T(X)-U(X)\| \leqslant M$ for each $X$ in $\mathrm{E}_{1}$. Then $\|T(r X)-r U(X)\| \leqslant M$ for each $r>0$ and hence

$$
\lim _{r \rightarrow \infty} r^{-1} T(r X)=U(X) \text { for each } X \text { in } \mathbf{E}_{1} .
$$

It follows that the only positive homogeneous (hence the only linear) isometry candidate for $U$ uniformly near $T$ is given by (1.3). (Note that if the limit in (1.3) exists for each $X$ in $\mathbf{E}_{1}$ then the map $U$ so defined is in fact a positive homogeneous isometry.) In general given an $\epsilon$-isometry $T: \mathbf{E}_{1} \rightarrow \mathbf{E}_{2}$ the isometry $U$ associated with $T$ by (1.3) (assuming it exists) need not be linear. It remains an open question whether $U$ must be linear if $T$ is surjective. However, for $T$ surjective and $\operatorname{dim} E_{1}=\operatorname{dim} E_{2}$ (the case of main concern in this paper) it is true that the associated isometry $U$ is, whenever it exists, linear. In fact, any isometry between two finite dimensional spaces of the same dimension which takes 0 to 0 is linear. (To see this observe that the range of such a map must be closed since its domain is complete, and the range must be open by invariance of domain since an isometry is a homeomorphism. Hence the isometry must be surjective and the Mazur-Ulam theorem [1, p. 166]-which states that an isometry from one normed linear space onto another which transforms 0 to 0 must be linear-applies to yield the desired conclusion. That invariance of domain is relevant to this type of argument was first pointed out to me by Peter L. Renz.) It also follows from the above remark that given $T: \mathbf{E}_{1} \rightarrow \mathrm{E}_{2}$ $\left(\operatorname{dim} \mathrm{E}_{1}=\operatorname{dim} \mathrm{E}_{2}\right)$ and an isometry $U$ such that, for some $M \geqslant 0$, $\| T(X)-$ $U(X) \| \leqslant M$ for each $X$ in $\mathbf{E}_{1}$ then $U-U(0)$ is linear and thus given by (1.3).

A summary of these results is contained in

Proposition 1.4. Suppose that $\operatorname{dim} \mathbf{E}_{1}=\operatorname{dim} \mathbf{E}_{2}$ and $T: \mathbf{E}_{1} \rightarrow \mathbf{E}_{2}$ is a surjective $\epsilon$-isometry with $T(0)=0$. A necessary condition that $(1.1)$ hold is that $U(X)=\lim _{r \rightarrow \infty} r^{-1} T(r X)$ exists for each $X$ in $\mathbf{E}_{1}$. If $U$ does exist then it is a linear isometry of $\mathbf{E}_{1}$ onto $\mathbf{E}_{2}$ and is the only possible isometry taking 0 to 0 which uniformly approximates $T$.

Before turning to the statements and proofs of the main results, some preliminary remarks are in order. The notation $\left.\left(r_{i}\right)\right\rceil \infty$ means: $\left(r_{i}\right)_{i=1}^{\infty}$ is a sequence of positive numbers increasing strictly monotonically to $\infty$.

REMARKS 1.5. (a) If $\operatorname{dim} \mathbf{E}_{1}<\operatorname{dim} \mathbf{E}_{2}$ then isometries from $\mathbf{E}_{1}$ into $\mathbf{E}_{2}$ which take 0 to 0 need not be positive homogeneous, let alone linear. Indeed, let $\mathbf{Q} \subset \ell_{1}^{2}$ denote the positive quadrant and call a function $f: \mathbf{R} \rightarrow \ell_{1}^{2}$ monotone 
increasing if whenever $t_{1}<t_{2}$, then $f\left(t_{2}\right)-f\left(t_{1}\right) \in \mathbf{Q}$. Let $f$ be any monotone increasing continuous function such that $f(0)=(0,0)$. For any number $t$ let $U(t)=f(g(t))$ where $g(t)$ is the unique number with the properties $\|f(g(t))\|=$ $|t|$ and $\operatorname{tg}(t) \geqslant 0$. Because the norm of $\ell_{1}^{2}$ is additive on $\mathbf{Q}$ it is easy to check that $U$ is an isometry of $\mathbf{R}$ into $\ell_{1}^{2}$ which sends 0 to $(0,0)$.

(b) If $\mathbf{E}_{\mathbf{2}}$ is a strictly convex Banach space then any isometry $U$ of $\mathbf{E}_{\mathbf{1}}$ into $E_{2}$ which transforms 0 to 0 must be homogeneous. (This follows directly from the strict triangle inequality that would obtain supposing the contrary.) Consequently when $\mathbf{E}_{2}$ is strictly convex and $T: \mathbf{E}_{1} \rightarrow \mathbf{E}_{2}$ is a surjective $\epsilon$-isometry, the only possible isometry $U$ uniformly close to $T$ is given by (1.3). Moreover if $\|T(X)-U(X)\| \leqslant M$ for each $X$ in $\mathbf{E}_{1}$ then $U-U(0)$ is linear. Indeed, it suffices to prove $U\left(\mathbf{E}_{1}\right)$ is dense in $\mathbf{E}_{2}$. (Since $U\left(\mathbf{E}_{1}\right)$ is closed in $\mathbf{E}_{2}$, it will follow that $U-U(0)$ is surjective and hence, by the Mazur-Ulam theorem, linear.) For any $Y$ in $\mathrm{E}_{2}$ and for each positive integer $n$, pick any point $X_{n} \in T^{-1}(n Y)$. Then $\left\|U\left(X_{n}\right)-n Y\right\| \leqslant M$ or, using the fact that $U$ is positive homogeneous, $\lim _{n \rightarrow \infty} U\left(n^{-1} X_{n}\right)=Y$.

(c) Suppose that $\mathbf{E}_{2}$ is a finite dimensional Banach space and that $T$ : $\mathbf{E} \rightarrow \mathrm{E}_{2}$. Then for each $\left.\left(r_{i}\right)\right\rceil \infty$ and $X$ in $\mathbf{E}$ there is a cluster point of $\left(r_{i}^{-1} T\left(r_{i} X\right)\right)_{i=1}^{\infty}$. Moreover all cluster points of this type have norm equal to $\|X\|$. (Indeed,

$$
\lim _{i \rightarrow \infty}\left\|r_{i}^{-1} T\left(r_{i} X\right)\right\|=\lim _{i \rightarrow \infty} r_{i}^{-1}\left[r_{i}\|X\|+\theta_{i} \epsilon\right]=\|X\|
$$

so that $\left(r_{i}^{-1} T\left(r_{i} X\right)\right)_{i=1}^{\infty}$ is a bounded sequence in $\mathbf{E}_{2}$, a finite dimensional space.)

(d) Suppose that $T: \mathbf{E} \rightarrow \mathrm{E}^{\prime}, X \in \mathrm{E}, Y \in \mathrm{E}^{\prime}$, and $\left.\left(r_{i}\right)\right\rceil \infty$. Then $\lim _{i \rightarrow \infty} r_{i}^{-1} T\left(r_{i} X\right)=Y$ if and only if $\lim _{i \rightarrow \infty} r_{i}^{-1} S\left(r_{i} Y\right)=X$. In fact,

$$
\begin{aligned}
\lim _{i \rightarrow \infty}\left\|r_{i}^{-1} S\left(r_{i} Y\right)-X\right\| & =\lim _{i \rightarrow \infty} r_{i}^{-1}\left[\left\|S\left(r_{i} Y\right)-S T\left(r_{i} X\right)\right\|+\theta_{i} \epsilon\right] \\
& =\lim _{i \rightarrow \infty}\left\|Y-r_{i}^{-1} T\left(r_{i} X\right)\right\| .
\end{aligned}
$$

DEFinition 1.6. Given $\delta \geqslant 0$ a subset $\mathbf{A}$ of a Banach space $\mathbf{E}$ is said to be $\delta$-onto if for each $X$ in $\mathbf{E}$ there is a point $Y$ in $\mathrm{A}$ with $\|X-Y\| \leqslant \delta$. A function whose range lies in a Banach space is called $\delta$-onto if its range is $\delta$-onto.

Although subsequent results are stated for surjective $\epsilon$-isometries observe that only minor alterations are needed to adjust the proofs if 'surjective' is replaced by ' $\delta$-onto for some $\delta<\infty$ '.

2. It is important in light of Proposition 1.4 to show that $\lim _{r \rightarrow \infty} r^{-1} T(r X)$ exists whenever $T$ is a surjective $\epsilon$-isometry between two finite dimensional Banach spaces. This problem is tackled in several steps, the final results being listed in Theorem 2.7. 
THEOREM 2.2. For any two finite dimensional Banach spaces $\mathbf{E}_{1}$ and $\mathbf{E}_{2}$ and surjective $\in$-isometry $T: \mathbf{E}_{1} \rightarrow \mathbf{E}_{2}$ for which $T(0)=0$ suppose that $V, W \in$ $\mathbf{E}_{1}, V^{\prime}, W^{\prime} \in \mathbf{E}_{2}$, and $\left(n_{i}\right) \uparrow \infty$ satisfy:

$$
\lim _{i \rightarrow \infty} n_{i}^{-1} T\left(n_{i} V\right)=V^{\prime} \text { and } \lim _{i \rightarrow \infty} n_{i}^{-1} T\left(n_{i} W\right)=W^{\prime} .
$$

Then

$$
\lim _{i \rightarrow \infty} n_{i}^{-1} T\left(n_{i}(1 / 2 V+1 / 2 W)\right)=1 / 2 V^{\prime}+1 / 2 W^{\prime} .
$$

The proof of this theorem is modeled on that of the Mazur-Ulam theorem $[1$, p. 166] although, since we are dealing with approximate rather than true isometries, it is necessary to 'push to infinity' to properly mimic their proof.

NotAtion 2.3. (a) Let

$$
\begin{aligned}
& \mathbf{H}_{0}=\{V, W\} ; \quad \mathbf{H}_{0}^{\prime}=\left\{V^{\prime}, W^{\prime}\right\} ; \\
& \mathbf{H}_{1}=\left\{Z \in \mathbf{E}_{1} \mid\|V-Z\|=\|W-Z\|=1 / 2\|V-W\|\right\} ; \\
& \mathbf{H}_{1}^{\prime}=\left\{Z \in \mathbf{E}_{2} \mid\left\|V^{\prime}-Z\right\|=\left\|W^{\prime}-Z\right\|=1 / 2\left\|V^{\prime}-W^{\prime}\right\|\right\} ;
\end{aligned}
$$

and inductively for each integer $l>1$ define

$$
\begin{aligned}
& \mathbf{H}_{l}=\left\{Z \in \mathbf{H}_{l-1} \mid\|Z-X\| \leqslant 1 / 2 \text { diameter } \mathbf{H}_{l-1} \text { for each } X \in \mathbf{H}_{l-1}\right\}, \\
& \mathbf{H}_{l}^{\prime}=\left\{Z \in \mathbf{H}_{l-1}^{\prime} \mid\|Z-X\| \leqslant 1 / 2 \text { diameter } \mathbf{H}_{l-1}^{\prime} \text { for each } X \in \mathbf{H}_{l-1}^{\prime}\right\} .
\end{aligned}
$$
$\left(t_{k}\right)_{k=1}^{\infty}$.

(b) The notation $\left(s_{h}\right)_{h=1}^{\infty} \subset\left(t_{k}\right)_{k=1}^{\infty}$ means: $\left(s_{h}\right)_{h=1}^{\infty}$ is a subsequence of

As will become evident in the proof of Lemma 2.5 , it is necessary for technical reasons to determine that diameter $\mathbf{H}_{l}=$ diameter $\mathbf{H}_{l}^{\prime}$ for every $l \geqslant 0$.

LEMMA 2.4. Let $X \in \mathrm{H}_{l}$ and suppose that $X^{\prime}=\lim _{j \rightarrow \infty} r_{j}^{-1} T\left(r_{j} X\right)$ exists where $\left(r_{j}\right)_{j=1}^{\infty} \subset\left(n_{i}\right)_{i=1}^{\infty}$. Then $X^{\prime} \in \mathbf{H}_{l}^{\prime}$. Conversely, if $Y^{\prime} \in \mathbf{H}_{l}^{\prime}$ and $\lim _{j \rightarrow \infty} r_{j}^{-1} S\left(r_{j} Y^{\prime}\right)=Y$ exists, then $Y \in \mathbf{H}_{l}$. Consequently diameter $\mathbf{H}_{l}=$ diameter $\mathbf{H}_{l}^{\prime}$ for each $l \geqslant 0$.

Proof. The proof is by induction on $l$, the case $l=0$ following from the definitions of $\mathrm{H}_{0}$ and $\mathrm{H}_{0}^{\prime}, 1.5(\mathrm{~d})$ and the fact that

$$
\left\|V^{\prime}-W^{\prime}\right\|=\lim _{i \rightarrow \infty}\left\|n_{i}^{-1} T\left(n_{i} V\right)-n_{i}^{-1} T\left(n_{i} W\right)\right\|=\|V-W\| .
$$

The case $l=1$ should be treated separately, but since the proof is similar to the general inductive step for $l>1$ we omit it.

Thus assume that $l>1$ and that the lemma is established for $l-1$. For any $X$ in $\mathbf{H}_{l}$ and $\left(r_{j}\right)_{j=1}^{\infty} \subset\left(n_{i}\right)_{i=1}^{\infty}$ for which $X^{\prime}=\lim _{j \rightarrow \infty} r_{j}^{-1} T\left(r_{j} X\right)$ exists we wish to show $X^{\prime} \in \mathbf{H}_{l}^{\prime}$. Pick any $Z^{\prime} \in \mathbf{H}_{l-1}^{\prime}$ and find $\left(p_{h}\right)_{h=1}^{\infty} \subset\left(r_{j}\right)_{j=1}^{\infty}$ such that $Z=$ 
$\lim _{h \rightarrow \infty} p_{h}^{-1} S\left(p_{h} Z^{\prime}\right)$ exists. By the inductive hypothesis $Z \in \mathbf{H}_{l-1}$. Moreover, as is easily checked $\|X-Z\|=\left\|X^{\prime}-Z^{\prime}\right\|$. Then $\|X-Z\| \leqslant 1 / 2$ diameter $\mathbf{H}_{l-1}$ since $X \in \mathrm{H}_{l}$ and $Z \in \mathrm{H}_{l-1}$ so that by the inductive hypothesis, $\left\|X^{\prime}-Z^{\prime}\right\| \leqslant$ $1 / 2$ diameter $\mathbf{H}_{l-1}^{\prime}$ for each $Z^{\prime} \in \mathbf{H}_{l-1}^{\prime}$. To conclude that $X^{\prime} \in \mathbf{H}_{l}^{\prime}$ it thus suffices to show that $X^{\prime} \in \mathbf{H}_{l-1}^{\prime}$. But since $X \in \mathbf{H}_{l} \subset \mathbf{H}_{l-1}$ the inductive hypothesis forces $X^{\prime} \in \mathbf{H}_{l-1}^{\prime}$ which completes half the inductive step. Since the other half is similar it is omitted.

Finally, to check that the diameters of $\mathbf{H}_{l}$ and $\mathbf{H}_{l}^{\prime}$ are the same it suffices by symmetry to show that diameter $\mathbf{H}_{l} \leqslant$ diameter $\mathbf{H}_{l}^{\prime}$. But if $X, Y \in \mathbf{H}_{l}$ pick $\left(r_{j}\right)_{j=1}^{\infty} \subset\left(n_{i}\right)_{i=1}^{\infty}$ such that both the following limits exist: $\lim _{j \rightarrow \infty} r_{j}^{-1} T\left(r_{j} X\right)=X^{\prime}$ and $\lim _{j \rightarrow \infty} r_{j}^{-1} T\left(r_{j} Y\right)=Y^{\prime}$. Evidently $\left\|X^{\prime}-Y^{\prime}\right\|=\|X-Y\|$ and since $X^{\prime}, Y^{\prime} \in$ $\mathrm{H}_{l}^{\prime}$ from above, the lemma follows.

The next lemma forms the backbone of the proof of Theorem 2.2 and is analogous in form to that in which $U$ is a surjective isometry between two normed spaces. The statement corresponding to Lemma 2.5 would read: $U\left(H_{l}\right)=$ $\mathbf{H}_{l}^{\prime}$ for each $l \geqslant 0$.

LEMMA 2.5. For any $\delta>0$ and integer $l \geqslant 0$ there is a number $M(\delta, l)$ such that if $n_{i} \geqslant M(\delta, l)$ then

(1) $n_{i}^{-1} T\left(n_{i} X\right) \in \mathrm{H}_{l}^{\prime}+\mathrm{B}_{2}(0, \delta)$ for each $X$ in $\mathrm{H}_{l}$; and

(2) $n_{i}^{-1} S\left(n_{i} X\right) \in \mathrm{H}_{l}+\mathrm{B}_{1}(0, \delta)$ for each $X$ in $\mathrm{H}_{l}^{\prime}$.

Proof. The proof is by induction on $l$. The $l=0$ case follows directly from 1.5(d). As in Lemma 2.4, despite the fact that the case $l=1$ should be treated separately, verification of this step is almost exactly the same as the first part of the proof of the general inductive step, and is consequently omitted. Assume then that $l>1$ and that for each $\alpha>0$ a number $M(\alpha, l-1)$ satisfying the conditions of Lemma 2.5 has been determined.

Choose any number $M_{l}$ such that

$$
\left\{X \in \mathrm{H}_{l-1}+\mathbf{B}_{1}\left[0, M_{l}^{-1}\right] \mid\|X-Z\| \leqslant 1 / 2 \text { diameter } \mathbf{H}_{l-1}+M_{l}^{-1}\right. \text { for each }
$$

(a)

$$
\left.Z \in \mathbf{H}_{l-1}\right\} \subset \mathbf{H}_{l}+\mathbf{B}_{1}(0, \delta) \text { and }
$$

(I)

$$
\left\{X \in \mathbf{H}_{l-1}^{\prime}+\mathbf{B}_{2}\left[0, M_{l}^{-1}\right] \mid\|X-Z\| \leqslant \not / 2 \text { diameter } \mathbf{H}_{l-1}^{\prime}+M_{l}^{-1}\right. \text { for each }
$$

(b)

$$
\left.Z \in \mathbf{H}_{l-1}^{\prime}\right\} \subset \mathbf{H}_{l}^{\prime}+\mathbf{B}_{2}(0, \delta) .
$$

(To see that such a number exists observe first that if $\mathbf{A}_{\boldsymbol{n}}$ denotes the set on the left side of ' $C$ ' in (I)(a) above with $M_{l}$ replaced by $n$, then $A_{n}$ is compact for 
each integer $n, A_{n} \supset A_{n+1}$, and $\bigcap_{n=1}^{\infty} A_{n}=H_{l}$. Hence $A_{n} \subset H_{l}+B_{1}(0, \delta)$ for some $n$ since $\mathrm{H}_{l}+\mathrm{B}_{1}(0, \delta)$ is an open set containing $\mathrm{H}_{l}$. A similar argument works for the sets in (b), and taking the larger of the two $n$ 's so obtained produces one choice of $M_{l}$.) Let

$$
M(\delta, l)=\max \left\{M\left(\left(2 M_{l}\right)^{-1}, l-1\right), 12 \in M_{l}\right\} .
$$

It remains to verify the conclusion of the lemma for this choice. Thus assume $n_{i} \geqslant M(\delta, l)$. The case presented here-corresponding to (2) of 2.5 -is similar to that for (1); thus that part of the inductive step corresponding to a verification of (1) of Lemma 2.5 is omitted.

For any points $X$ in $\mathbf{H}_{l}^{\prime}$ and $Z$ in $\mathbf{H}_{l-1}$ choose $Y$ in $\mathbf{E}_{2}$ so that

$$
\left\|S\left(n_{i} Y\right)-n_{i} Z\right\| \leqslant \epsilon .
$$

The first step is to show that $Y$ is close to $\mathbf{H}_{l-1}^{\prime}$ (cf. (VI)). Applying $T$ to (III) yields $\left\|n_{i} Y-T\left(n_{i} Z\right)\right\| \leqslant 2 \epsilon$ or, in more convenient form,

$$
\left\|Y-n_{i}^{-1} T\left(n_{i} Z\right)\right\| \leqslant 2 \epsilon n_{i}^{-1} .
$$

Because $n_{i} \geqslant M\left(\left(2 M_{l}\right)^{-1}, l-1\right)$ and $Z \in \mathrm{H}_{l-1}$ it follows that

$$
n_{i}^{-1} T\left(n_{i} Z\right) \in \mathrm{H}_{l-1}^{\prime}+\mathrm{B}_{2}\left(0,\left(2 M_{l}\right)^{-1}\right) \text {. }
$$

Combining (IV) and (V) yields

$$
Y \in \mathrm{H}_{l-1}^{\prime}+\mathrm{B}_{2}\left(0,\left(2 M_{l}\right)^{-1}+2 \epsilon n_{i}^{-1}\right) .
$$

Our goal is to show that $n_{i}^{-1} S\left(n_{i} X\right)$ is an element of the left side (hence of the right side) of (I)(a). Observe that

$$
\begin{aligned}
\left\|n_{i}^{-1} S\left(n_{i} X\right)-Z\right\| & =n_{i}^{-1}\left\|S\left(n_{i} X\right)-n_{i} Z\right\| \\
& \leqslant n_{i}^{-1}\left[\left\|S\left(n_{i} X\right)-S\left(n_{i} Y\right)\right\|+\epsilon\right] \quad \text { (from (III)) } \\
& \leqslant\|X-Y\|+2 \epsilon n_{i}^{-1} .
\end{aligned}
$$

Recall that $X \in \mathbf{H}_{l}^{\prime}$. Hence (VI) yields $\|X-Y\| \leqslant 1 / 2$ diameter $\mathbf{H}_{l-1}^{\prime}+\left(2 M_{l}\right)^{-1}+$ $2 \epsilon n_{i}^{-1}$ which then combined with (VII) gives

$$
\left\|n_{i}^{-1} S\left(n_{i} X\right)-Z\right\| \leqslant 1 / 2 \text { diameter } \mathbf{H}_{l-1}^{\prime}+\left(2 M_{l}\right)^{-1}+4 \epsilon n_{i}^{-1}
$$

$$
\leqslant 1 / 2 \text { diameter } \mathbf{H}_{l-1}+M_{l}^{-1} \text { (from Lemma } 2.4 \text { and (Ii)). }
$$

Moreover from $X \in \mathrm{H}_{l}^{\prime} \subset \mathrm{H}_{l-1}^{\prime}$ and (II) evidently

$$
n_{i}^{-1} S\left(n_{i} X\right) \in \mathrm{H}_{l-1}+\mathrm{B}_{1}\left(0,\left(2 M_{l}\right)^{-1}\right) \subset \mathrm{H}_{l-1}+\mathrm{B}_{1}\left[0, M_{l}^{-1}\right] \text {. }
$$

Combining (VIII) which holds for each $Z$ in $\mathbf{H}_{l-1}$, (IX), and (I)(a) yields the 
desired conclusion: $n_{i}^{-1} S\left(n_{i} X\right) \in \mathrm{H}_{l}+\mathrm{B}_{1}(0, \delta)$ whenever $X \in \mathrm{H}_{l}^{\prime}$ and $n_{i} \geqslant$ $M(\delta, l)$. From the remarks made at the beginning of the inductive step, this completes the proof of Lemma 2.5 .

Proof of Theorem 2.2. The idea is to demonstrate that whenever $\delta>$ 0 and $l \geqslant 3$ is an integer, then $n_{i}^{-1} T\left(n_{i}(1 / 2 V+1 / 2 W)\right) \in \mathbf{H}_{l}^{\prime}+\mathbf{B}_{2}(0, \delta)$ for sufficiently large $n_{i}$. Because $\bigcap_{n=1}^{\infty} \mathbf{H}_{n}^{\prime}=\left\{1 / 2 V^{\prime}+1 / 2 W^{\prime}\right\}$ and diameter $\mathbf{H}_{n+1}^{\prime} \leqslant$ $1 / 2$ diameter $\mathbf{H}_{n}^{\prime}$ for each $n$ (cf. [1, pp. 166-167]) it will follow that $\lim _{i \rightarrow \infty} n_{i}^{-1} T\left(n_{i}(1 / 2 V+1 / 2 W)\right)$ exists and equals $1 / 2 V^{\prime}+1 / 2 W^{\prime}$.

Fix $\delta>0$ and suppose that $n_{i} \geqslant M(\delta, l)$. For any $Z$ in $\mathrm{H}_{l-1}^{\prime}$ find $Y$ in $E_{1}$ such that

$$
T\left(n_{i} Y\right)=n_{i} Z
$$

(which is possible since $T$ is surjective). Note that

$$
Y \in \mathrm{H}_{l-1}+\mathrm{B}_{1}\left(0,\left(2 M_{l}\right)^{-1}+\epsilon n_{i}^{-1}\right)
$$

as can be seen as follows: $S T\left(n_{i} Y\right)=S\left(n_{i} Z\right)$ from (1) so that

$$
\left\|Y-n_{i}^{-1} S\left(n_{i} Z\right)\right\| \leqslant \epsilon n_{i}^{-1}
$$

From $Z \in \mathrm{H}_{l-1}^{\prime}$ coupled with $n_{i} \geqslant M\left(\left(2 M_{l}\right)^{-1}, l-1\right)$ we obtain $n_{i}^{-1} S\left(n_{i} Z\right) \in$ $\mathbf{H}_{l-1}+\mathbf{B}_{1}\left(0,\left(2 M_{l}\right)^{-1}\right)$ which combined with (3) yields (2).

We next show that

$$
\left\|n_{i}^{-1} T\left(n_{i}(1 / 2 V+1 / 2 W)\right)-Z\right\| \leqslant 1 / 2 \text { diameter } \mathbf{H}_{l-1}^{\prime}+M_{l}^{-1}
$$

$$
\text { for each } Z \in \mathbf{H}_{l-1}^{\prime} \text {. }
$$

Indeed

$$
\begin{aligned}
\left\|n_{i}^{-1} T\left(n_{i}(1 / 2 V+1 / 2 W)\right)-Z\right\|=n_{i}^{-1}\left\|T\left(n_{i}(1 / 2 V+1 / 2 W)\right)-T\left(n_{i} Y\right)\right\| \\
\quad \leqslant\|1 / 2 V+1 / 2 W-Y\|+\epsilon n_{i}^{-1} \\
\quad \leqslant 1 / 2 \text { diameter } \mathbf{H}_{l-1}+\left(2 M_{l}\right)^{-1}+\epsilon n_{i}^{-1}+\epsilon n_{i}^{-1} .
\end{aligned}
$$

(Note that $1 / 2 V+1 / 2 W \in \bigcap_{n=1}^{\infty} \mathbf{H}_{n}$ and hence is in $\mathbf{H}_{l}$. This latter inequality now follows from (2).) Line (4) follows directly using Lemma 2.4 and the definition of $M(\delta, l)$.

Finally, since $1 / 2 V+1 / 2 W \in \mathbf{H}_{l-1}$ and $n_{i} \geqslant M\left(\left(2 M_{l}\right)^{-1}, l-1\right)$ it follows that

(5) $n_{i}^{-1} T\left(n_{i}(1 / 2 V+1 / 2 W)\right) \in \mathbf{H}_{l-1}^{\prime}+\mathbf{B}_{2}\left(0,\left(2 M_{l}\right)^{-1}\right) \subset \mathbf{H}_{l-1}^{\prime}+\mathbf{B}_{2}\left[0, M_{l}^{-1}\right]$. 
Combining (4), (5) and the definition of $M_{l}$ yields $n_{i}^{-1} T\left(n_{i}(1 / 2 V+1 / 2 W)\right) \in$ $\mathbf{H}_{l}^{\prime}+\mathbf{B}_{2}(0, \delta)$ and from the earlier remarks this completes the proof of Theorem 2.2.

Theorem 2.7 below gives some conditions on a finite dimensional Banach space $\mathbf{E}$ under which $\lim _{r \rightarrow \infty} r^{-1} T(r X)$ exists for each $X$ in $\mathbf{E}$ and surjective $T: \mathbf{E} \rightarrow \mathbf{E}$. Although it seems likely that the finite dimensionality of $\mathbf{E}$ is enough to guarantee the existence of this limit (cf. also 4.3) our results are more modest. It is convenient to introduce the following

Notation 2.6. For a finite dimensional Banach space $\mathbf{E}$ with closed unit ball B denote by ex B the set of extreme points of B with the relative topology. Moreover, for $X$ in ex B let $\mathrm{K}(X)$ refer to the component (relative to ex B) of $X$, and let $\mathrm{D}(\mathrm{E})=\{X \in \operatorname{ex} \mathbf{B} \mid \mathbf{K}(X)=\{X\}\}$. Finally let $E=\{\mathbf{E} \mid \mathbf{E}$ is a finite dimensional Banach space and linear span $D(E)=E$. (Note that linear span $\mathrm{D}(\mathrm{E})=\mathrm{E}$ if and only if each $Y$ in $\mathrm{E}$ can be written in the form $Y=\Sigma_{j=1}^{l} r_{j} Y_{j}$ with $Y_{j} \in \mathrm{D}(\mathrm{E})$ and $r_{j} \geqslant 0$ for each $j$ since $\mathrm{D}(\mathrm{E})$ is symmetric about 0 .)

Observe that the elements of $E$ evidently properly include those finite dimensional spaces with polyhedral unit balls and, more generally, those for which the set of extreme points of the unit ball is a totally disconnected topological space.

THEOREM 2.7. Let $\mathbf{E}_{1}$ and $\mathbf{E}_{2}$ be finite dimensional spaces such that either $\mathbf{E}_{1}$ or $\mathbf{E}_{2}$ belongs to $E$. Suppose that $T: \mathbf{E}_{1} \rightarrow \mathrm{E}_{2}$ is a surjective $\epsilon-$ isometry with $T(0)=0$. Then $\lim _{r \rightarrow \infty} r^{-1} T(r X)=U(X)$ exists for each $X$ in $\mathbf{E}_{1}$. It follows that both $\mathbf{E}_{1}$ and $\mathbf{E}_{2}$ are in $E$. (Indeed $U$ is a linear isometry between them.) Moreover $\lim _{\|X\| \rightarrow \infty}\|X\|^{-1}\|T(X)-U(X)\|=0$.

Our method of proof requires three preparatory lemmas, the first of which roughly states that the behavior of an $\epsilon$-isometry is almost continuous.

LEMMA 2.8 (P. L. RENZ). Let $\mathbf{N}$ and $\mathbf{N}^{\prime}$ be normed linear spaces and $T: \mathbf{N} \rightarrow \mathbf{N}^{\prime}$ an $\epsilon$-isometry such that $T(0)=0$. Then there is a continuous $4 \epsilon-$ isometry $T^{*}: \mathrm{N} \rightarrow \mathrm{N}^{\prime}$ such that $\left\|T^{*}(X)-T(X)\right\| \leqslant 2 \epsilon$ for each $X$ in $\mathbf{N}$.

Proof. Consider all subsets of $\mathbf{N}$ with the property that the distance between each pair of distinct elements is at least $\epsilon / 8$. By Zorn's lemma there is a maximal such collection which is denoted henceforth by $\left\{X_{\gamma}\right\}_{\gamma \in \Gamma}$. Note that for any $X$ in $\mathrm{N}$ there is a $\gamma$ in $\Gamma$ with $\left\|X-X_{\gamma}\right\| \leqslant \epsilon / 8$.

Let $\mathrm{B}_{\gamma}$ be shorthand notation for $\mathrm{B}\left(X_{\gamma}, \epsilon / 4\right)$. Then $\left\{\mathrm{B}_{\gamma} \mid \gamma \in \Gamma\right\}$ is an open cover of $\mathbf{N}$ and hence there is a partition of unity $\left\{f_{\xi}\right\}_{\xi \in \Xi}$ subordinate to $\left\{\mathbf{B}_{\gamma}\right\}_{\gamma \in \Gamma}$. For each $\xi \in \Xi$ pick any $\gamma$ in $\Gamma$ such that $\left\{Y \in \mathbf{N} \mid f_{\xi}(Y) \neq 0\right\} \subset \mathbf{B}_{\gamma}$ and denote this $\gamma$ by the symbol $\gamma(\xi)$. (Thus in the new notation $\left\{Y \in N \mid f_{\xi}(Y) \neq\right.$ 
$0\} \subset B_{\gamma(\xi)}$ for each $\xi$ in $\Xi$.) Define $T^{*}: \mathbf{N} \rightarrow N^{\prime}$ by the formula

$$
T^{*}(X)=\sum_{\xi \in \Xi} f_{\xi}(X) T\left(X_{\gamma(\xi)}\right) \quad \text { for each } X \text { in N. }
$$

It is standard that the function $T^{*}$ is well defined and continuous. Moreover for $X$ in $\mathbf{N}$ we have

$$
\begin{aligned}
\| T^{*}(X) & -T(X)\|=\| \sum_{\xi \in \Xi} f_{\xi}(X)\left[T\left(X_{\gamma(\xi)}\right)-T(X)\right] \| \\
& \leqslant \sum_{\xi \in \Xi} f_{\xi}(X)\left\|T\left(X_{\gamma(\xi)}\right)-T(X)\right\| \leqslant \sum_{\xi \in \Xi} f_{\xi}(X)\left[\left\|X_{\gamma(\xi)}-X\right\|+\epsilon\right] .
\end{aligned}
$$

But if $f_{\xi}(X) \neq 0$ then $\left\|X-X_{\gamma(\xi)}\right\|<\epsilon / 4$ so that

$$
\left\|T^{*}(X)-T(X)\right\| \leqslant \sum_{\xi \in \Xi} f_{\xi}(X)[\epsilon / 4+\epsilon]=5 \epsilon / 4
$$

for each $X$ in $\mathbf{N}$. Because $T$ is an $\epsilon$-isometry the above inequality leads directly to the conclusion that $T^{*}$ is a $\left(3 \frac{1}{2}\right) \epsilon-$ (hence a $4 \epsilon-$ ) isometry. This completes the proof of Lemma 2.8 .

As noted in 1.5(c) whenever $E_{2}$ is finite dimensional, $T: \mathbf{E} \rightarrow \mathbf{E}_{2}$, and $X \in \mathrm{E}$ then for each sequence $\left(r_{i}\right) / \infty$ the sequence $\left(r_{i}^{-1} T\left(r_{i} X\right)\right)_{i=1}^{\infty}$ has a cluster point.

Notation 2.9. Let $\mathrm{Cl}_{T}(X)$ denote the set of all such cluster points as $\left(r_{i}\right) \uparrow \infty$ ranges. $\left(\mathrm{Cl}_{T}(X)\right.$ will be referred to as the cluster set for $T$ at $X$.)

Evidently $\mathrm{Cl}_{T}(X) \subset\left\{Y \in \mathrm{E}_{2} \mid\|Y\|=\|X\|\right\}$. Some other properties of this set which will be applied in the proof of Theorem 2.7 are given by

Lemma 2.10. Let $\mathbf{E}$ be a normed linear space, $\mathbf{E}_{2}$ a finite dimensional Banach space and $T: \mathbf{E} \rightarrow \mathbf{E}_{2}$ an $\epsilon$-isometry such that $T(0)=0$. Then for each $X$ in $\mathrm{E}$ the set $\mathrm{Cl}_{T}(X)$ is nonempty, compact, and connected.

Proof. It is straightforward to check that $\mathbf{C l}_{T}(X)$ is closed, which in light of the above discussion shows that $\mathrm{Cl}_{T}(X)$ is nonempty and compact. If $\mathrm{Cl}_{T}(X)=\mathrm{C}_{1} \cup \mathrm{C}_{2}$ with $\mathbf{C}_{1} \cap \mathbf{C}_{2}=\varnothing$ and each $\mathbf{C}_{i}, i=1,2$, a closed subset of $\mathrm{Cl}_{T}(X)$, then $\mathbf{C}_{1}$ and $\mathbf{C}_{2}$ are closed and disjoint in $\mathbf{E}_{2}$ so that there are open sets $\mathrm{O}_{1}$ and $\mathbf{O}_{2}$ in $\mathrm{E}_{2}$ with $\mathrm{O}_{1} \cap \mathrm{O}_{2}=\varnothing$ and $\mathrm{O}_{i} \supset \mathrm{C}_{i}, i=1,2$.

Choose a continuous $4 \epsilon$-isometry $T^{*}: \mathrm{E} \rightarrow \mathrm{E}_{2}$ such that $\left\|T^{*}(X)-T(X)\right\| \leqslant$ $2 \epsilon$ for each $X$ in $\mathbf{E}$ (cf. 2.8) and note that $\mathrm{Cl}_{T^{*}}(X)=\cdot \mathrm{Cl}_{T}(X)$. (Indeed $Y \in$ $\mathrm{Cl}_{T}(X)$ if and only if there is a sequence $\left.\left(r_{i}\right)\right\rceil \infty$ such that $\lim _{i \rightarrow \infty} r_{i}^{-1} T\left(r_{i} X\right)=$ $Y$, if and only if there is a sequence $\left(r_{i}\right) / \infty$ with $\lim _{i \rightarrow \infty} r_{i}^{-1} T^{*}\left(r_{i} X\right)=Y$ since

$$
\left\|r_{i}^{-1} T\left(r_{i} X\right)-r_{i}^{-1} T^{*}\left(r_{i} X\right)\right\| \leqslant 2 \epsilon r_{i}^{-1} \rightarrow 0 \text { as } i \rightarrow \infty,
$$

if and only if $Y \in \mathrm{Cl}_{T^{*}}(X)$.) 
Find $N>0$ such that whenever $r \geqslant N$ then $r^{-1} T^{*}(r X) \in 0_{1} \cup O_{2}$. (Such an $N$ exists since otherwise there is a sequence $\left(r_{i}\right) / \infty$ with $r_{i}^{-1} T^{*}\left(r_{i} X\right) \notin 0_{1} \cup$ $\mathbf{O}_{2}$ for each $i$. But since $\left(r_{i}^{-1} T^{*}\left(r_{i} X\right)\right)_{i=1}^{\infty}$ has a cluster point-which is hence an element of $\mathrm{Cl}_{T^{*}}(X)=\mathrm{Cl}_{T}(X) \subset \mathrm{O}_{1} \cup \mathrm{O}_{2}$-it follows that $r_{i}^{-1} T^{*}\left(r_{i} X\right) \in 0_{1} \cup$ $\mathbf{O}_{\mathbf{2}}$ infinitely often.)

Let $\mathbf{A}_{i}=\left\{r \geqslant N \mid r^{-1} T^{*}(r X) \in \mathbf{0}_{i}\right\}, i=1,2$. Then $\mathbf{A}_{1} \cup \mathbf{A}_{2}=[N, \infty)$, $\mathbf{A}_{1} \cap \mathbf{A}_{2}=\varnothing$, and each $\mathbf{A}_{i}$ is an open subset of $[N, \infty)$ since the map $r \rightarrow$ $r^{-1} T^{*}(r X)$ is continuous and each $\mathbf{O}_{i}$ is open. Since $[N, \infty)$ is connected, either $\mathbf{A}_{1}$ or $\mathbf{A}_{2}$-hence either $\mathbf{C}_{1}$ or $\mathbf{C}_{2}$-is empty. Thus $\mathbf{C l}_{T}(X)$ is connected, which completes the proof of Lemma 2.10 .

Lemma 2.11. Let $\mathbf{E}_{1}$ and $\mathbf{E}_{2}$ be finite dimensional Banach spaces and $T$ : $\mathbf{E}_{1} \rightarrow \mathbf{E}_{2}$ a surjective e-isometry such that $T(0)=0$. For $X$ in $\mathbf{E}_{1}$ and $Y$ in $\mathbf{E}_{2}$ each of norm 1 suppose $\lim _{i \rightarrow \infty} r_{i}^{-1} T\left(r_{i} X\right)=Y$ for some sequence $\left(r_{i}\right) / \infty$. Then $X \in \operatorname{exB}_{1}[0,1]$ if and only if $Y \in \operatorname{exB}_{2}[0,1]$.

Proof. Because $\lim _{i \rightarrow \infty} r_{i}^{-1} T\left(r_{i} X\right)=Y$ if and only if $\lim _{i \rightarrow \infty} r_{i}^{-1} S\left(r_{i} Y\right)=$ $X$ the statement of Lemma 2.11 is almost symmetric in $X$ and $Y$. The only stumbling block is that $S$ need not be surjective. However $S$ is $\epsilon$-onto as noted in 1.2(f) (2) so that Theorem 2.2 may be applied to this map (cf. the remarks after Definition 1.6). Since the only result needed in the proof of either direction of this lemma is Theorem 2.2, the statement of Lemma 2.11 is in effect symmetric in $X$ and $Y$, and it thus suffices to demonstrate that $X \notin \operatorname{exB}_{1}[0,1]$ implies that $Y \notin \operatorname{exB}_{2}[0,1]$. If $X=1 / 2 X_{1}+1 / 2 X_{2}$ where $\left\|X_{1}\right\|=\left\|X_{2}\right\|=1$ and $\| X_{1}-$ $X_{2} \|>0$ find $\left(n_{j}\right)_{j=1}^{\infty} \subset\left(r_{i}\right)_{i=1}^{\infty}$ such that $\lim _{j \rightarrow \infty} n_{j}^{-1} T\left(n_{j} X_{i}\right)=Y_{i}$ exists for $i=$ 1,2. Then $\left\|Y_{i}\right\|=\left\|X_{i}\right\|=1$ and $\left\|Y_{1}-Y_{2}\right\|=\left\|X_{1}-X_{2}\right\|>0$. Moreover, it follows from Theorem 2.2 that

$$
Y=\lim _{j \rightarrow \infty} n_{j}^{-1} T\left(n_{j} X\right)=\lim _{j \rightarrow \infty} n_{j}^{-1} T\left(n_{j}\left(1 / 2 X_{1}+1 / 2 X_{2}\right)\right)=1 / 2 Y_{1}+1 / 2 Y_{2}
$$

so that $Y$ is not an extreme point, finishing the proof.

Before proceeding to a proof of Theorem 2.7 observe that Lemma 2.11 shows that $X \in \operatorname{exB}_{1}[0,1]$ if and only if $\mathrm{Cl}_{T}(X) \subset \operatorname{exB}_{2}[0,1]$ (and symmetrically, $Y \in \operatorname{exB}_{2}[0,1]$ if and only if $\left.\mathrm{Cl}_{S}(Y) \subset \operatorname{exB}_{1}[0,1]\right)$.

Proof OF THEOREM 2.7. Because all subsequent arguments are in effect symmetric in $\mathbf{E}_{1}$ and $\mathbf{E}_{2}$ assume without loss of generality that $\mathbf{E}_{2} \in E$. For any point $Y_{0} \in \operatorname{exB}_{2}[0,1]$ pick any sequence $\left.\left(r_{i}\right)\right\rceil \infty$ such that $X_{0}=$ $\lim _{i \rightarrow \infty} r_{i}^{-1} S\left(r_{i} Y_{0}\right)$ exists. Then $X_{0} \in \operatorname{exB}_{1}[0,1]$ (cf. Lemma 2.11) and hence $\mathrm{Cl}_{T}\left(X_{0}\right) \subset \operatorname{exB}_{2}[0,1]$ (2.11 again). Moreover if $Y_{0} \in \mathrm{D}\left(\mathrm{E}_{2}\right)$ then since $\mathrm{Cl}_{T}\left(X_{0}\right)$ is connected (cf. Lemma 2.10) it follows that $\lim _{r \rightarrow \infty} r^{-1} T\left(r X_{0}\right)$ exists and equals $Y_{0}$. (Also, for any $\alpha>0$, evidently $\lim _{r \rightarrow \infty} r^{-1} T\left(r \alpha X_{0}\right)$ exists and equals $\alpha Y_{0}$.) 
Note that when $Z_{1}, \ldots, Z_{l}$ are any points of $\mathrm{E}_{1}$ such that $\lim _{r \rightarrow \infty} r^{-1} T\left(r Z_{i}\right)=$ $W_{i}$ exists for each $i=1, \ldots, l$, then Theorem 2.2 shows that $\lim _{r \rightarrow \infty} r^{-1} T\left(r \Sigma_{i=1}^{l} Z_{i}\right)$ exists and is $\Sigma_{i=1}^{l} W_{i}$. Since $\mathrm{D}\left(\mathrm{E}_{2}\right)$ positively generates $\mathrm{E}_{2}$, given any point $Y$ in $\mathrm{E}_{2}$, find $Y_{1}, \ldots, Y_{l}$ in $\mathrm{D}\left(\mathrm{E}_{2}\right)$ and $r_{1}, \ldots, r_{l} \geqslant 0$ such that $\Sigma_{i=1}^{l} r_{i} Y_{i}=Y$. Then find $X_{1}, \ldots, X_{l}$ in $\mathbf{E}_{1}$ (as above) such that $\lim _{r \rightarrow \infty} r^{-1} T\left(r X_{i}\right)$ exists and equals $Y_{i}$ for each $i$. Let $X=\Sigma_{i=1}^{l} r_{i} X_{i}$. Then $\lim _{r \rightarrow \infty} r^{-1} T(r X)$ exists and equals

$$
\lim _{r \rightarrow \infty} r^{-1} T\left(r \sum_{i=1}^{l} r_{i} X_{i}\right)=\sum_{i=1}^{l} r_{i} Y_{i}=Y .
$$

In order to show that $\lim _{r \rightarrow \infty} r^{-1} T(r X)$ exists for each $X$ in $\mathbf{E}_{1}$, pick any sequence $\left(r_{i}\right) \uparrow \infty$ such that $Y=\lim _{i \rightarrow \infty} r_{i}^{-1} T\left(r_{i} X\right)$ exists. Then there is a point $X_{1} \in \mathrm{E}_{1}$ from above such that $\lim _{r \rightarrow \infty} r^{-1} T\left(r X_{1}\right)$ exists and equals $Y$. But this means that $\lim _{r \rightarrow \infty} r^{-1} S(r Y)=X_{1}$. On the other hand, from the definition of $Y$, $\lim _{i \rightarrow \infty} r_{i}^{-1} S\left(r_{i} Y\right)=X$. Consequently $X=X_{1}$ and hence $\lim _{r \rightarrow \infty} r^{-1} T(r X)$ exists (and equals $Y$ ). From Proposition 1.4 it follows that $U$ (as defined in the statement of Theorem 2.7) is a linear isometry from $\mathbf{E}_{\mathbf{1}}$ onto $\mathbf{E}_{\mathbf{2}}$ and hence $\mathbf{E}_{1} \in E$.

It remains to show that $\lim _{\|X\| \rightarrow \infty}\|X\|^{-1}\|T(X)-U(X)\|=0$. If not, there is a sequence $\left(X_{l}\right)_{l=1}^{\infty}$ in $\mathbf{E}_{1}$ and a sequence $\left.\left(n_{l}\right)\right\rceil \infty$ for which:

(a) $\left\|X_{l}\right\|=1$ for each $l$;

(b) $\lim _{l \rightarrow \infty} X_{l}=X_{0}$ exists; and

(c) $\lim \inf _{l \rightarrow \infty}\left\|n_{l} X_{l}\right\|^{-1}\left\|T\left(n_{l} X_{l}\right)-U\left(n_{l} X_{l}\right)\right\|>0$.

But since $\lim _{l \rightarrow \infty} n_{l}^{-1} T\left(n_{l} X_{l}\right)=U\left(X_{0}\right)$ (which follows easily from (b)) it follows that the lim inf in (c) can be rewritten in the form:

$$
\liminf _{l \rightarrow \infty}\left\|n_{l}^{-1} T\left(n_{l} X_{l}\right)-U\left(X_{l}\right)\right\|=0 .
$$

This contradiction completes the proof of Theorem 2.7.

3. A special case of Theorem 2.7 states that for each surjective $\epsilon$-isometry $T: \ell_{1}^{k} \rightarrow \ell_{1}^{k}$ with $T(0)=0$ there is an isometry $U$ (given by (1.3)) for which $\|T(X)-U(X)\|=o(\|X\|)$. Theorem 3.1 strengthens this result by providing a positive answer to (1.1) for $E_{1}=E_{2}=\ell_{1}^{k}$.

THEOREM 3.1. For each positive integer $k$ there is a constant $K$ such that whenever $T: \ell_{1}^{k} \rightarrow \ell_{1}^{k}$ is a surjective $\epsilon$-isometry with $T(0)=0$ there is a (linear) isometry $U: \ell_{1}^{k} \rightarrow \ell_{1}^{k}$ satisfying $\|T(X)-U(X)\| \leqslant K \epsilon$ for each $X$ in $\ell_{1}^{k}$. We may take $K=100 k^{3}-50 k^{2}+25 k+3$.

The proof splits naturally into two parts, the first of which is to demonstrate the result when $X$ is restricted to being a multiple of a coordinate vector (cf. 
(3.6)). The general conclusion follows from the first step together with an elementary 'interpolation' device (cf. 3.7).

Notation 3.2. (a) Write $B$ instead of $B[0,1]$ and let $\mathbf{B}^{2 k-1}$ denote the set of all $2 k-1$ tuples of points of $\mathbf{B}$ (considered as a subset of the $(2 k-1)$ fold product of $\ell_{1}^{k}$ ).

(b) Throughout this section

$V_{i}=(0, \ldots, 0,1,0, \ldots, 0)$ and $V_{k+i}=(0, \ldots, 0,-1,0, \ldots, 0)$ where the only nonzero entry occurs in the $i$ th place, $i=1, \ldots, k$.

LEMMA 3.3. For a point $Y_{0}=\left(y_{1}, \ldots, y_{k}\right) \in \ell_{1}^{k}$ of norm one suppose that $\left|y_{i_{0}}\right| \geqslant\left|y_{j}\right|$ for $j \neq i_{0}$. Then for every point $\left(Y_{1}, \ldots, Y_{2 k-1}\right) \in \mathrm{B}^{2 k-1}$ it follows that

$$
\sum_{0 \leqslant i<j \leqslant 2 k-1}\left\|Y_{i}-Y_{j}\right\| \leqslant 4 k^{2}-2 k-2\left(1-v_{i_{0}} \mid\right) .
$$

Moreover there is a point of $\mathrm{B}^{2 k-1}$ at which this inequality becomes equality.

Proof. Since a linear isometry of $\ell_{1}^{k}$ simply interchanges some of the coordinates and multiplies others by -1 it suffices to prove the lemma when $y_{1} \geqslant y_{2} \geqslant \cdots \geqslant y_{k} \geqslant 0$. Define the function $f\left[Y_{0}\right]: \mathbf{B}^{2 k-1} \rightarrow \mathbf{R}$ by: $f\left[Y_{0}\right]\left(Y_{1}, \ldots, Y_{2 k-1}\right)=\Sigma_{0 \leqslant i<j \leqslant 2 k-1}\left\|Y_{i}-Y_{j}\right\|$. Since $f\left[Y_{0}\right]$ is a continuous convex function on the compact convex set $\mathbf{B}^{2 k-1}$ it attains its maximum on $\operatorname{exB}^{2 k-1}$. (Observe that $\left(Y_{1}, \ldots, Y_{2 k-1}\right) \in \operatorname{exB}^{2 k-1}$ if and only if $\left\{Y_{i}\right\}_{i=1}^{2 k-1} \subset$ $\left\{V_{i}\right\}_{i=1}^{2 k}$.)

It remains to compute the maximum value of $f\left[Y_{0}\right]$ on $\operatorname{exB}^{2 k-1}$. Suppose first that $\left(Y_{1}, \ldots, Y_{2 k-1}\right) \in \operatorname{exB}^{2 k-1}$ has two or more of the $Y_{j}$ 's equal. Then

$$
\begin{array}{r}
f\left[Y_{0}\right]\left(Y_{1}, \ldots, Y_{2 k-1}\right)=\sum_{i=1}^{2 k-1}\left\|Y_{0}-Y_{i}\right\|+\sum_{1<i<j \leqslant 2 k-1}\left\|Y_{i}-Y_{j}\right\| \\
\leqslant 2(2 k-1)+2((k-1)(2 k-1)-1)=4 k^{2}-2 k-2 .
\end{array}
$$

On the other hand, if all the $Y_{i}$ 's in $\left(Y_{1}, \ldots, Y_{2 k-1}\right)$ are distinct extreme points of $\mathrm{B}$, because $\left\|Y_{0}-V_{1}\right\| \leqslant\left\|Y_{0}-V_{j}\right\|$ for $j=2, \ldots, 2 k$ it follows that

$$
\begin{aligned}
\sum_{i=1}^{2 k-1}\left\|Y_{0}-Y_{i}\right\| & \leqslant \sum_{i=2}^{2 k}\left\|Y_{0}-V_{i}\right\|=\sum_{i=2}^{k}\left\|Y_{0}-V_{i}\right\|+\sum_{i=k+1}^{2 k}\left\|Y_{0}-V_{i}\right\| \\
& =\sum_{i=2}^{k}\left[1-y_{i}+\sum_{j \neq i ; j=1}^{k} y_{j}\right]+2 k=\sum_{i=2}^{k}\left[2-2 y_{i}\right]+2 k \\
& =2\left(k-1-\left(1-y_{1}\right)\right)+2 k=4 k-4+2 y_{1} .
\end{aligned}
$$


Hence

$$
\begin{aligned}
& f\left[Y_{0}\right]\left(Y_{1}, \ldots, Y_{2 k-1}\right) \leqslant f\left[Y_{0}\right]\left(V_{2}, \ldots, V_{2 k}\right) \\
& =4 k-4+2 y_{1}+\sum_{2 \leqslant i<j<2 k}\left\|V_{i}-V_{j}\right\|=4 k^{2}-2 k-2\left(1-y_{1}\right)
\end{aligned}
$$

which completes the proof.

In order to prove Theorem 3.1 it is sufficient to prove that such a $K$ exists when the $\epsilon$-isometries $T$ under consideration are further restricted so that $U(X)=$ $\lim _{r \rightarrow \infty} r^{-1} T(r X)=X$ for each $X$ in $\ell_{1}^{k}$. This simplifying assumption about $U$ will henceforth be made for the remainder of $\S 3$.

Notation 3.4. For $r>_{\epsilon}$ let $W_{i}(r)=r^{-1} T\left(r V_{i}\right)$ for $i=1, \ldots, 2 k$. Then $W_{i}(r) \neq 0$ and define $X_{i}(r)=W_{i}(r) /\left\|W_{i}(r)\right\|$.

Lemma 3.5. Suppose that $r \geqslant 5 \epsilon\left[10 k^{2}-5 k+2\right]$. Then for integers $i$ and $j$ between 1 and $2 k$ we have

$$
\left\|W_{j}(r)-V_{i}\right\| \leqslant r^{-1} \epsilon\left(10 k^{2}-5 k+2\right) \text { if and only if } i=j .
$$

PROof. The following properties of $W_{i}(r)$ and $X_{i}(r)$ are easily established:

(a) $\left|\left\|W_{i}(r)\right\|-1\right| \leqslant \epsilon r^{-1}$;

(b) $\left\|W_{i}(r)-W_{n}(r)\right\| \geqslant 2-\epsilon r^{-1}$ for $1 \leqslant i<n \leqslant 2 k$;

(c) $\left\|X_{i}(r)\right\|=1$ for $i=1, \ldots, 2 k$;

(d) $\left\|X_{i}(r)-W_{i}(r)\right\| \leqslant 2 \epsilon r^{-1}$ for $i=1, \ldots, 2 k$;

(e) $\left\|X_{i}(r)-X_{n}(r)\right\| \geqslant 2-5 \epsilon r^{-1}$ for $1 \leqslant i<n \leqslant 2 k$;

(f) $\Sigma_{1<i<n \leqslant 2 k}\left\|X_{i}(r)-X_{n}(r)\right\| \geqslant 4 k^{2}-2 k-5 \epsilon r^{-1}\left(2 k^{2}-k\right)$.

Fix $j$ between 1 and $2 k$ and write $X_{j}(r)=\left(x_{1}(r), \ldots, x_{k}(r)\right)$. Then pick $i_{0}$ such that $\left|x_{i_{0}}(r)\right| \geqslant\left|x_{n}(r)\right|$ for $n \neq i_{0}$. As a direct consequence of Lemma 3.3 and (f) above

$$
\begin{aligned}
4 k^{2} & -2 k-2\left(1-\left|x_{i_{0}}(r)\right|\right) \\
& \geqslant f\left[X_{j}(r)\right]\left(X_{1}(r), \ldots, X_{j-1}(r), X_{j+1}(r), \ldots, X_{2 k}(r)\right) \\
& \geqslant 4 k^{2}-2 k-5 \epsilon r^{-1}\left(2 k^{2}-k\right)
\end{aligned}
$$

so that

(g) $2\left(1-\left|x_{i_{0}}(r)\right|\right) \leqslant 5 \epsilon r^{-1}\left(2 k^{2}-k\right)$.

Write $w_{j}(r)=\left(w_{1}(r), \ldots, w_{k}(r)\right)$ and let $V\left(i_{0}\right)=\left(\operatorname{sgn} w_{i_{0}}(r)\right) V_{i_{0}}$. Note that from (d), the restrictions on $r$, and the fact that $\left|x_{i_{0}}(r)\right| \geqslant k^{-1}$ since it is the largest of the absolute values of the coordinates of $X_{j}(r)$, a point of norm one, it follows that $w_{i_{0}}(r) \neq 0$ and that sgn $w_{i_{0}}(r)=\operatorname{sgn} x_{i_{0}}(r)$. Combining this with (g) yields 


$$
\left\|W_{j}(r)-V\left(i_{0}\right)\right\| \leqslant\left\|W_{j}(r)-X_{j}(r)\right\|+\left\|X_{j}(r)-\left(\operatorname{sgn} w_{i_{0}}(r)\right) V_{i_{0}}\right\|
$$

$$
\begin{aligned}
& \leqslant 2 \epsilon r^{-1}+1-\left|x_{i_{0}}(r)\right|+\sum_{i \neq i_{0} ; i=1}^{k}\left|x_{i}(r)\right|=2 \epsilon r^{-1}+2\left(1-\left|x_{i_{0}}(r)\right|\right) \\
& \leqslant r^{-1} \epsilon\left(10 k^{2}-5 k+2\right) .
\end{aligned}
$$

From $\epsilon r^{-1}\left(10 k^{2}-5 k+2\right) \leqslant 1 / 5$ and $(\mathrm{h})$ together with the fact that $V\left(i_{0}\right) \in\left\{V_{i}\right\}_{i=1}^{2 k}$ it follows that for each $r$ under consideration there is a unique $i$ between 1 and $2 k$ such that $\left\|W_{j}(r)-V_{i}\right\| \leqslant \epsilon r^{-1}\left(10 k^{2}-5 k+2\right)$. To emphasize the possible dependence of this integer $i$ on $r$ denote it temporarily by $i(r)$. It remains to show that $i(r)=j$ for each appropriate $r$.

Pick any $n \geqslant 5 \epsilon\left(10 k^{2}-5 k+2\right)$ and suppose $n \leqslant p \leqslant n+\epsilon$. We will show that $i(n)=i(p)$. Indeed

$$
\begin{aligned}
\left\|W_{j}(p)-W_{j}(n)\right\| & \leqslant n^{-1}\left\|T\left(p V_{j}\right)-T\left(n V_{j}\right)\right\|+\left(n^{-1}-p^{-1}\right)\left\|T\left(p V_{j}\right)\right\| \\
& \leqslant 2 \epsilon n^{-1}+\epsilon(n p)^{-1}(p+\epsilon) \leqslant 4 \epsilon n^{-1} \leqslant 1 / 5
\end{aligned}
$$

Consequently

$$
\begin{aligned}
& \left\|V_{i(p)}-V_{i(n)}\right\| \\
& \quad \leqslant\left\|V_{i(p)}-W_{j}(p)\right\|+\left\|W_{j}(p)-W_{j}(n)\right\|+\left\|W_{j}(n)-V_{i(n)}\right\| \leqslant 3 / 5 .
\end{aligned}
$$

Since $\left\|V_{i(p)}-V_{i(n)}\right\|$ is either 0 or 2 it follows that $i(n)=i(p)$ whenever $|p-n| \leqslant$ $\epsilon$, and hence $i(r)$ is independent of $r$ for $r \geqslant 5 \epsilon\left(10 k^{2}-5 k+2\right)$.

Finally, recall that $\lim _{r \rightarrow \infty} r^{-1} T\left(r V_{j}\right)=U\left(V_{j}\right)=V_{j}$ by the standing hypothesis. Otherwise written, this amounts to the statement $\lim _{r \rightarrow \infty}\left\|W_{j}(r)-V_{j}\right\|=0$ which shows that $i(r)=j$ for sufficiently large $r$ and hence for all $r \geqslant$ $5 \epsilon\left(10 k^{2}-5 k+2\right)$. This completes the proof of Lemma 3.5.

When $0 \leqslant r<5 \epsilon\left(10 k^{2}-5 k+2\right)$ we have

$$
\left\|T\left(r V_{j}\right)-r V_{j}\right\| \leqslant\left\|T\left(r V_{j}\right)\right\|+r \leqslant 2 r+\epsilon \leqslant \epsilon\left(100 k^{2}-50 k+21\right) .
$$

Combining this with the conclusion of Lemma 3.5 yields

$$
\left\|T\left(r V_{j}\right)-r V_{j}\right\| \leqslant \epsilon\left(100 k^{2}-50 k+21\right)
$$

for each $r \geqslant 0$ and $j=1, \ldots, 2 k$.

As indicated after the statement of Theorem 3.1, (3.6) marks the end of the first step of the proof of 3.1. The second step is much more direct.

Lemma 3.7. Suppose $X \in \ell_{1}^{k}$ and $M \geqslant 0$. Denote $\|X\|$ by the letter $r$ and 
suppose that $Y \in \ell_{1}^{k}$ is any point such that both $\|Y\| \leqslant r$ and \|\|$Y-r V_{j} \|-$ $\left\|X-r V_{j}\right\| \mid \leqslant M$ for $j=1, \ldots, 2 k$. Then $\|X-Y\| \leqslant k M$.

Proof. Write $X=\left(x_{1}, \ldots, x_{k}\right)$ and $Y=\left(y_{1}, \ldots, y_{k}\right)$. Observe that for each $j=1, \ldots, k$ we have $r \geqslant\left|x_{j}\right|$ and $r \geqslant\left|y_{j}\right|$ so that rewriting the $j$ th and $(j+k)$ th inequalities occurring in the statement of the lemma in terms of coordinates yields:

$$
r-y_{j}+\sum_{i \neq j ; i=1}^{k}\left|y_{i}\right|-\left[r-x_{j}+\sum_{i \neq j ; i=1}^{k}\left|x_{i}\right|\right]=\theta_{j} M
$$

and

$$
r+y_{j}+\sum_{i \neq j ; i=1}^{k}\left|y_{i}\right|-\left[r+x_{j}+\sum_{i \neq j ; i=1}^{k}\left|x_{i}\right|\right]=\theta_{j+k} M .
$$

Combine and simplify these two equations to get $\left|v_{j}-x_{j}\right| \leqslant M$ for $j=1, \ldots, k$. Hence $\|Y-X\|=\sum_{j=1}^{k}\left|y_{j}-x_{j}\right| \leqslant k M$. Q.E.D.

Proof of Theorem 3.1. Pick any $X$ in $\ell_{1}^{k}$ and let $r$ denote $\|X\|$. If $r \leqslant$ $2 \epsilon$ then $\|T(X)-X\| \leqslant\|T(X)\|+2 \epsilon \leqslant 5 \epsilon \leqslant \epsilon\left(100 k^{3}-50 k^{2}+25 k+3\right)$. Assume then that $r>2 \epsilon$. Let $Y=r T(X) /\|T(X)\|$. Then $\|Y-T(X)\| \leqslant 3 \epsilon$. Hence for each $j=1, \ldots, 2 k$ we have

$$
\begin{aligned}
\left\|Y-r V_{j}\right\| & =\left\|T(X)-r V_{j}\right\|+3 \epsilon \theta_{1} \\
& =\left\|T(X)-T\left(r V_{j}\right)\right\|+3 \epsilon \theta_{1}+\theta_{2}\left\|T\left(r V_{j}\right)-r V_{j}\right\| \\
& =\left\|X-r V_{j}\right\|+\epsilon \theta_{3}+3 \epsilon \theta_{1}+\theta_{4} \epsilon\left(100 k^{2}-50 k+21\right)
\end{aligned}
$$

by (3.6). That is

$$
\|\| Y-r V_{j}\|-\| X-r V_{j} \| \mid \leqslant \epsilon\left(100 k^{2}-50 k+25\right) .
$$

When Lemma 3.7 is applied $\|Y-X\| \leqslant \epsilon\left(100 k^{3}-50 k^{2}+25 k\right)$ results and since $\|Y-T(X)\| \leqslant 3 \epsilon$ we have $\|T(X)-X\| \leqslant \epsilon\left(100 k^{3}-50 k^{2}+25 k+3\right)$ for each $X$ in $\ell_{1}^{k}$. The proof of Theorem 3.1 is thus complete.

4. We begin this section with some remarks concerning surjectivity. As previously mentioned, with substantially the same proofs each result of $\S \S 1-3$ which concerns surjective $\epsilon$-isometries remains valid if 'surjective' is replaced by the somewhat more relaxed condition ' $\delta$-onto for some $\delta<\infty$ '. Peter L. Renz conjectured that a continuous $\epsilon$-isometry between two finite dimensional spaces of the same dimension is necessarily surjective. In fact, much more is true:

Proposition 4.1 (Dallas Webster). Let $E_{1}, E_{2}$ be $n$-dimensional Banach spaces and $h: E_{1} \rightarrow E_{2}$ a continuous, nonsurjective map with 
$\lim _{\|X\| \rightarrow \infty}\|h(X)\|=\infty$. Then given $M>0$ there are points $X, Y \in E_{1}$ with $\|X-Y\| \geqslant M$ and $h(X)=h(Y)$.

Proof. It evidently suffices to prove the result for $E_{1}=E_{2}=\mathrm{E}^{n}$, Euclidean $n$-space. Given $M$ define $s: \mathbf{E}^{n} \rightarrow S^{n}-\{p\}$ ( $p$ the north pole) to be the usual sterographic projection with the $n-1$ sphere centered at the origin of radius $M$ sent to the equator of $S^{n}$. Define $\widetilde{h}: S^{n} \rightarrow S^{n}$ by: (1) $\widetilde{h}(p)=p$ and (2) $\tilde{h}(q)=\operatorname{shs}^{-1}(q)$ for each $q \in S^{n}-\{p\}$. Since $\tilde{h}$ is not surjective there is a homeomorphism $k$ : range $\tilde{h} \rightarrow \mathrm{E}^{n}$, and since $k \tilde{h}: S^{n} \rightarrow \mathrm{E}^{n}$ is continuous, two antipodal points of $S^{n}$, say $a$ and $b$, are identified under $k \tilde{h}$ by the BorsukUlam antipodal mapping theorem [5, p. 349]. Evidently neither $a$ nor $b$ is $p$ and hence from $k \widetilde{h}(a)=k s\left(h s^{-1}(a)\right)=k s\left(h s^{-1}(b)\right)$ and the fact that $k s$ is one to one, we conclude $h s^{-1}(a)=h s^{-1}(b)$. Observe finally that by construction of $s$, $\left\|s^{-1}(a)-s^{-1}(b)\right\| \geqslant M$ whenever $a$ and $b$ are antipodal.

A close examination of the proof of Lemma 2.8 in conjunction with Proposition 4.1 shows that whenever $T: E_{1} \rightarrow E_{2}$ is an $\epsilon$-isometry and $\operatorname{dim} E_{1}=$ $\operatorname{dim} E_{2}$ then $T$ is $\epsilon$-onto (and obvious examples demonstrate that no stronger result is valid).

Turning now to the question of the validity of (1.1) in general, the following conjectures appear reasonable:

Conjecture 4.2. (1.1) has an affirmative answer whenever $\mathbf{E}_{1}$ or $\mathbf{E}_{2}$ is finite dimensional.

CONJECTURE 4.3. There is a separable Banach space $\mathbf{E}\left(=\mathbf{E}_{1}=\mathbf{E}_{2}\right)$ for which (1.1) has a negative answer.

The next results indicate a possible line of attack for the construction of a Banach space solving 4.3.

Proposition 4.4. Let $\mathbf{E}_{1}$ and $\mathbf{E}_{2}$ be separable Banach spaces and $\mathbf{A}_{i} \subset$ $\mathbf{E}_{i}, i=1,2$, subsets of the first category. Suppose that there are numbers $\delta_{i}, i=1,2$, such that $\mathbf{A}_{i}$ is $\delta_{i}$-onto. If $T^{\prime}: \mathbf{A}_{1} \rightarrow \mathbf{A}_{2}$ is a surjective map for which there is an $\epsilon^{\prime}>0$ satisfying $\left|\left\|T^{\prime}(V)-T^{\prime}(W)\right\|-\|V-W\|\right| \leqslant \epsilon^{\prime}$ for each $V, W$ in $\mathbf{A}_{1}$ then there is an extension $T$ of $T^{\prime}$ to an $\epsilon$-isometry of $\mathbf{E}_{1}$ onto $\mathbf{E}_{2}$ for some $\epsilon<\infty$. (We may take $\epsilon=4 \delta_{1}+2 \delta_{2}+\epsilon^{\prime}$.)

Proof. Assign to each $X$ in $\mathbf{E}_{1} \backslash \mathbf{A}_{1}$ a point $X_{1}$ in $\mathbf{A}_{1}$ with $\left\|X-X_{1}\right\| \leqslant \delta_{1}$ and define $T_{0}: \mathbf{E}_{1} \rightarrow \mathrm{E}_{2}$ by

$$
T_{0}(X)= \begin{cases}T^{\prime}(X) & \text { if } X \in \mathrm{A}_{1}, \\ T^{\prime}\left(X_{1}\right) & \text { if } X \in \mathrm{E}_{\underline{1}} \backslash \mathbf{A}_{1} .\end{cases}
$$

Then $T_{0}$ is a $\left(2 \delta+\epsilon^{\prime}\right)$-isometry extension of $T^{\prime}$ with range $A_{2}$. 
Observe that $\operatorname{card}\left(0 \backslash \mathbf{A}_{i}\right)=c, i=1,2$, whenever $\varnothing \neq 0$ is an open set in $\mathbf{E}_{i}$. Here 'card' denotes the cardinality of the set and $c$ is the cardinality of the continuum. (In fact $\mathbf{O} \backslash \mathbf{A}_{\boldsymbol{i}}$ is completely metrizable since it is a $\mathbf{G}_{\delta}$ subset of $\mathbf{O}$, a completely metrizable space. Moreover $\mathbf{O} \backslash \mathbf{A}_{i}$ lacks isolated points. Hence it contains a homeomorph of the Cantor discontinuum [8, p. 445] so that $c \leqslant$ $\operatorname{card}\left(0 \backslash \mathbf{A}_{i}\right) \leqslant \operatorname{card} \mathbf{E}_{i} \leqslant c$.) In particular then, we may write $\mathbf{E}_{2} \backslash \mathbf{A}_{2}=\{W(\gamma) \mid$ $\gamma \in \Gamma$ \} where $\Gamma$ denotes the set of ordinals of cardinality less than $c$.

Inductively define points $X(\gamma)$ in $\mathbf{E}$ as follows: Choose a point $Y(0)$ in $\mathbf{A}_{2}$ such that $\|W(0)-Y(0)\| \leqslant \delta_{2}$ and find $V(0)$ in $A_{1}$ with $T_{0}(V(0))=Y(0)$. Pick any point $X(0)$ in $\mathrm{E}_{1} \backslash \mathrm{A}_{1}$ such that $\|X(0)-V(0)\| \leqslant \delta_{1}$.

If points $X(\gamma), \gamma<\gamma_{0} \in \Gamma$, have been chosen, find $Y\left(\gamma_{0}\right)$ in $A_{2}$ such that $\left\|W\left(\gamma_{0}\right)-Y\left(\gamma_{0}\right)\right\| \leqslant \delta_{2}$ and pick $V\left(\gamma_{0}\right)$ in $A_{1}$ with $T_{0}\left(V\left(\gamma_{0}\right)\right)=Y\left(\gamma_{0}\right)$. Then let $X\left(\gamma_{0}\right)$ be any point of $\mathbf{E}_{1} \backslash\left(\mathbf{A}_{1} \cup\left\{X(\gamma) \mid \gamma<\gamma_{0}\right\}\right)$ such that $\left\|X\left(\gamma_{0}\right)-V\left(\gamma_{0}\right)\right\| \leqslant$ $\delta_{1}$. Note that such a choice is in fact possible since

$$
\operatorname{card}\left(\mathbf{B}\left(V\left(\gamma_{0}\right), \delta_{1}\right) \backslash\left(\mathrm{A}_{1} \cup\left\{X(\gamma) \mid \gamma<\gamma_{0}\right\}\right)\right)=\operatorname{card}\left(\mathrm{B}\left(V\left(\gamma_{0}\right), \delta_{1}\right) \backslash \mathbf{A}_{1}\right)=c
$$

from above.

Observe that, for each $\gamma$ in $\Gamma,\|X(\gamma)-V(\gamma)\| \leqslant \delta_{1}$ for some point $V(\gamma)$ in $\mathbf{A}_{1}$ for which $\left\|W(\gamma)-T_{0}(V(\gamma))\right\| \leqslant \delta_{2}$. Define the map $T: \mathbf{E}_{1} \rightarrow \mathbf{E}_{2}$ by

$$
T(X)= \begin{cases}T_{0}(X) & \text { if } X \neq X(\gamma) \text { for each } \gamma \text { in } \Gamma, \\ W(\gamma) & \text { if } X=X(\gamma) \text { for some } \gamma \text { in } \Gamma .\end{cases}
$$

It is clear that $T$ is a surjective extension of $T^{\prime}$, so it remains to check that $T$ is a $\left(4 \delta_{1}+2 \delta_{2}+\epsilon^{\prime}\right)$-isometry. For $\gamma, \gamma^{\prime} \in \Gamma$

$$
\begin{aligned}
& \left\|T(X(\gamma))-T\left(X\left(\gamma^{\prime}\right)\right)\right\|=\left\|W(\gamma)-W\left(\gamma^{\prime}\right)\right\| \\
& \quad=\left\|T_{0}(V(\gamma))-T_{0}\left(V\left(\gamma^{\prime}\right)\right)\right\|+\theta_{1}\left[\left\|T_{0}(V(\gamma))-W(\gamma)\right\|+\left\|T_{0}\left(V\left(\gamma^{\prime}\right)\right)-W\left(\gamma^{\prime}\right)\right\|\right] \\
& \quad=\left\|V(\gamma)-V\left(\gamma^{\prime}\right)\right\|+\theta_{2}\left(2 \delta_{1}+\epsilon^{\prime}\right)+\theta_{3} 2 \delta_{2} \\
& \quad=\left\|X(\gamma)-X\left(\gamma^{\prime}\right)\right\|+\theta_{4} 2 \delta_{1}+\theta_{2}\left(2 \delta_{1}+\epsilon^{\prime}\right)+\theta_{3} 2 \delta_{2} .
\end{aligned}
$$

Hence

$$
\|\| T(X(\gamma))-T\left(X\left(\gamma^{\prime}\right)\right)\|-\| X(\gamma)-X\left(\gamma^{\prime}\right) \| \mid \leqslant 4 \delta_{1}+2 \delta_{2}+\epsilon^{\prime},
$$

and the other cases are still easier to check. Thus the proof is complete.

For any $\epsilon$-isometry $T: \mathbf{E}_{1} \rightarrow \mathrm{E}_{2}$ and $r>0$ the map $T_{r}$ defined by $T_{r}(X)=$ $r^{-1} T(r X)$ for each $X$ in $\mathbf{E}_{1}$ is an $r^{-1} \epsilon$-isometry, and in particular, $U_{r}$ is an isometry whenever $U$ is. It is also clear that $\|T(X)-U(X)\| \leqslant K \epsilon$ for each $X$ in $\mathbf{E}_{1}$ if and 
only if $\left\|T_{r}(X)-U_{r}(X)\right\| \leqslant K r^{-1} \epsilon$ for each $X$ in $\mathbf{E}_{1}$. Consequently whenever $\mathbf{E}_{1}$ and $E_{2}$ are Banach spaces for which (1.1) has an affirmative answer, then given $\epsilon>0$ the number

$\inf \left\{K \mid\right.$ whenever $T: \mathbf{E}_{1} \rightarrow \mathbf{E}_{2}$ is a surjective $\epsilon$-isometry

for which $T(0)=0$ there is an isometry $U$ with $\|T(X)-U(X)\| \leqslant K \epsilon$

for each $X$ in $\mathbf{E}_{1}$ \}

is independent of $\epsilon$ and will be denoted by $K\left(E_{1}, E_{2}\right)$.

Proposition 4.5. Suppose that $\left(\mathrm{E}_{j}\right)_{j=1}^{\infty}$ is a sequence of separable Banach spaces with $\lim _{j \rightarrow \infty} K\left(\mathbf{E}_{j}, \mathbf{E}_{j}\right)=\infty$. Then there is a separable Banach space $\mathbf{E}$ for which (1.1) fails provided the isometries $U$ considered are all positive homogeneous (and hence have the form (1.3)).

Proof. By going to a subsequence if necessary assume that $\lim _{j \rightarrow \infty} 2^{-i} K\left(\mathbf{E}_{j}, \mathbf{E}_{j}\right)=\infty$ and let $\mathbf{E}$ be the $\ell_{1}$ sum of the $\mathbf{E}_{j}$ 's. That is, $\mathbf{E}=$ $\left\{\left(X_{i}\right)_{i=1}^{\infty} \mid X_{j} \in \mathrm{E}_{j}\right.$ for each $j$ and $\left.\left\|\left(X_{i}\right)\right\|=\Sigma\left\|X_{i}\right\|<\infty\right\}$. Since there is nothing to prove if for some surjective $T: \mathbf{E} \rightarrow \mathbf{E}$ (or $T: \mathbf{E}_{j} \rightarrow \mathbf{E}_{j}$ for some $j$ ) and $X$ in $\mathbf{E}\left(X\right.$ in $\left.\mathrm{E}_{j}\right)$ the $\lim _{r \rightarrow \infty} r^{-1} T(r X)$ fails to exist, henceforth assume that under the above conditions such limits always exist.

For each $j$ choose a surjective $2^{-j}$-isometry $T_{j}: \mathbf{E}_{j} \rightarrow \mathrm{E}_{j}\left(\right.$ with $T_{j}(0)=0$ ) such that $\left\|T_{j}\left(X_{j}\right)-U_{j}\left(X_{j}\right)\right\| \geqslant 2^{-(j+1)} K\left(\mathrm{E}_{j}, \mathrm{E}_{j}\right)$ for some $X_{j}$, where $U_{j}$ is the isometry of form (1.3) associated with $T_{j}$. Moreover let $A_{1}=A_{2}=\bigoplus_{j=1}^{\infty} E_{j}$, so that $\mathbf{A}_{i}, i=1,2$, is a dense first category subset of $\mathbf{E}$. Define $T^{\prime}: \mathbf{A}_{1} \rightarrow \mathbf{A}_{2}$ by the formula

$$
T^{\prime}\left(Y_{1}, \ldots, Y_{n}, \ldots\right)=\left(T_{1}\left(Y_{1}\right), \ldots, T_{n}\left(Y_{n}\right), \ldots\right)
$$

and observe that since $T_{j}$ is surjective and $T_{j}(0)=0$ for each $j, T^{\prime}$ satisfies the conditions of Proposition 4.4 with $\epsilon^{\prime}=\Sigma 2^{-j}=1$. Let $T: \mathbf{E} \rightarrow \mathrm{E}$ be a surjective 2-isometry extension of $T^{\prime}$. Note that for any $Y=\left(Y_{1}, \ldots, Y_{n}, 0, \ldots\right) \in$ $A_{1}$ we have

$$
\begin{aligned}
\lim _{r \rightarrow \infty} r^{-1} T(r Y) & =\left(\lim _{r \rightarrow \infty} r^{-1} T_{1}\left(r Y_{1}\right), \ldots, \lim _{r \rightarrow \infty} r^{-1} T_{n}\left(r Y_{n}\right), 0, \ldots\right) \\
& =\left(U_{1}\left(Y_{1}\right), \ldots, U_{n}\left(Y_{n}\right), 0, \ldots\right)
\end{aligned}
$$

so that the isometry $U$ associated via (1.3) with $T$ is, by continuity, the map $U\left(\left(X_{i}\right)_{i=1}^{\infty}\right)=\left(U_{i}\left(X_{i}\right)\right)_{i=1}^{\infty}$ for each $\left(X_{i}\right)_{i=1}^{\infty}$ in E. But then Proposition 4.5 follows from

$$
\begin{aligned}
\sup \{\|T(X)-U(X)\| \mid X \in \mathrm{E}\} & \geqslant \sup \left\{\left\|T_{j}\left(X_{j}\right)-U_{j}\left(X_{j}\right)\right\| \mid j=1,2, \ldots\right\} \\
& \geqslant \sup \left\{2^{-(j+1)} K\left(\mathrm{E}_{j}, \mathrm{E}_{j}\right) \mid j=1,2, \ldots\right\}=\infty
\end{aligned}
$$

which completes the proof. 
Although an upper bound for $K\left(\ell_{1}^{j}, \ell_{1}^{j}\right)$ is established in Theorem 3.1 it is unknown if $\lim _{j \rightarrow \infty} K\left(\mathrm{E}_{j}, \mathrm{E}_{j}\right)=\infty$. Observe that $\ell_{1}$ provides a solution to Conjecture 4.3 (assuming all isometries are of the form (1.3)) if this limit is infinite since the Banach space $\mathbf{E}$ constructed in Proposition 4.5 for any subsequence of the sequence $\left(\ell_{1}^{j}\right)_{j=1}^{\infty}$ is $\ell_{1}$.

\section{REFERENCES}

1. S. Banach, Théorie des opérations linéaires, Monografie Mat., PWN, Warsaw, 1932; reprint, Chelsea, New York, 1955. MR 17, 175.

2. D. G. Bourgin, Approximate isometries, Bull. Amer. Math. Soc. 52 (1946), 704-714. MR 8, 157.

3. Approximately isometric and multiplicative transformations on continuous function rings, Duke Math. J. 16 (1949), 385-397. MR 11, 115.

4. Classes of transformations and bordering transformations, Bull. Amer. Math. Soc. 57 (1951), 223-237. MR 13, 138.

5. J. Dugundji, Topology, Allyn and Bacon, Boston, Mass., 1966. MR 33 \#1824.

6. D. H. Hyers and S. M. Ulam, On approximate isometries, Bull. Amer. Math. Soc. 51 (1945), 288-292. MR 7, 123.

7. - On approximate isometries of the space of continuous functions, Ann. of Math. 48 (1947), 285-289. MR 8, 588.

8. K. Kuratowski, Topology. Vol. I, PWN, Warsaw, 1958; English transl., Academic Press, New York, 1966. MR 19, 873; 36 \#440.

9. S. M. Ulam, $A$ collection of mathematical problems, Interscience Tracts in Pure and Appl. Math., no. 8, Interscience, New York and London, 1960. MR 22 \#10884.

DEPARTMENT OF MATHEMATICS, STATE UNIVERSITY OF NEW YORK, BUFFALO, NEW YORK 14222 